\title{
Heavy-Light Mesons and Chiral Symmetry
}

\author{
William A. Bardeen ${ }^{1}$ \\ ${ }^{1}$ Theoretical Physics, Fermilab, MS 106 \\ P.O.Box 500, Batavia, IL 60510, USA \\ bardeen@fnal.gov \\ (Dated: April 29, 2008)
}

\begin{abstract}
The chiral structure of heavy-light mesons is explored with a particular focus on the nature of the $D_{s J}$ charmed mesons. Theoretical predictions for the hadronic and radiative decays of these mesons are compared to recent experimental data.
\end{abstract}

PACS numbers: 11.30.Rd, 12.39.Fe, 12.39.Hg, 13.25.Ft, 14.40.Lb

\section{INTRODUCTION}

Chiral symmetry is a hidden symmetry associated with the existence of massless quarks. The simplest physical states containing light quarks are the heavy-light meson and baryons containing two heavy quarks. In the static limit for the heavy quarks, these systems define the physical states of a localized constituent, or tethered, quark. The recent discovery of narrow $D_{s J}$ mesons provides an opportunity to study directly the chiral structure of a single localized light quark.

In 2003, Babar[1] announced the discovery of a new narrow resonance decaying into $D_{s}+\pi^{0}$, the $D_{s J}(2317)$. This new state was novel because of its extremely narrow width and the low value of its mass. This discovery opened a new window on quantum chromodynamics, QCD, through the spectroscopy of heavy-light mesons, quarkonia and other new states involving heavy and light quarks.

Following the Babar announcement, CLEO[2] made a careful study of the $D_{s} \pi^{0} \gamma$ channel and announced the discovery of second narrow state, the $D_{s J}(2460)$, whose indications were already present in the Babar data. Belle[3] subsequently confirmed the existence of both new states and determined their quantum numbers to be $\left(0^{+}, 1^{+}\right)$by analyzing the angular distributions in the decays. Subsequently, many new states have been discovered by the B-factories and by CDF and DO at the Tevatron collider.

Before the discovery of the new states, most expectations for the spectroscopy of heavy-light mesons were based on potential models of a heavy quark bound to a constituent light quark [4-6]. Potential models did predict additional p-wave bound states with the quantum numbers of the new mesons but these states were expected to be heavy and decay rapidly to a D-meson and a K-meson. However, the new states discovered by Babar, CLEO and Belle were much lighter than potential model predictions, being more than $40 \mathrm{MeV}$ below the strong decay thresholds. There have been many subsequent interpretations of these new states and methods suggested to analyze their structure. These speculations include modified potential models, D-K molecules, fourquark models, hybrids, unitarized chiral meson bound states, bag models, QCD sum rules, phenomenological string models and lattice field theory.

Shortly after the Babar announcement, Estia Eichten, Chris Hill and I suggested[7] that the new Babar state should be the $0^{+}$member of a $D_{s J}\left(0^{+}, 1^{+}\right)$multiplet identified as the chiral partner of the $D_{s}\left(0^{-}, 1^{-}\right)$groundstate multiplet. We also made a prediction for the mass of the $1+$ state along with a detailed analysis of the decay modes of the new states that follows from this identification. The CLEO discovery confirmed our prediction for the mass of the new $1+$ state, and subsequent determinations of the branching ratios of various decay modes are largely in agreement with our predictions.

\section{SPECTROSCOPY AND STRONG DYNAMICS}

Heavy-light mesons may be viewed as the analogues of the hydrogen atom in atomic physics with a single light quark bound to a heavy core, the color-triplet heavy quark. In the static limit, the heavy quark spin decouples, and the states are classified by the total angular momentum of the light quark - gluon system. The groundstate of a $D_{s}$ meson has $J_{\text {light }}=1 / 2^{-}$while the new $D_{s J}$ states are interpreted as states with $J_{\text {light }}=1 / 2^{+}$.

As mentioned above, the new $D_{s J}$ states may be viewed as chiral partners of the $D_{s}$ ground-states whose dynamics are governed by the symmetries of the strong interactions including $S U(3)$ flavor symmetry, $S U(3) \times$ $S U(3) / S U(3)$ chiral symmetry and heavy quark symmetry. The $D_{s J}$ and $D_{s}$ ground-states form a chiral supermultiplet whose states would be degenerate in a chiralsymmetric phase of QCD. The splitting between the opposite parity states arises from the spontaneous breaking of the chiral symmetry, and the mass difference between the states is related to the magnitude of the chiral order parameter, $\Sigma$.

To implement a linear chiral symmetry multiplet structure in the HL sector we construct left-handed and righthanded linear combinations of the heavy spin multiplets. We define the following chiral combinations:

$$
\mathcal{H}_{L}=\frac{1}{\sqrt{2}}\left(\mathcal{H}-i \mathcal{H}^{\prime}\right) \quad \mathcal{H}_{R}=\frac{1}{\sqrt{2}}\left(\mathcal{H}+i \mathcal{H}^{\prime}\right)
$$


Under $S U(3)_{L} \times S U(3)_{R}$ these fields transform as $\mathcal{H}_{R} \sim$ $(1,3)$ and $\mathcal{H}_{L} \sim(3,1)$ respectively. We now write an effective Lagrangian involving both the HL mesons and the $\Sigma$ field, implementing HQ symmetry and chiral symmetry. The lowest order effective Lagrangian to first order in an expansion in the chiral field $\Sigma$, and to zeroth order in $\left(1 / m_{Q}\right)$ :

$$
\begin{gathered}
\mathcal{L}_{L H}=-i \frac{1}{2} \operatorname{Tr}\left(\overline{\mathcal{H}}_{L} v \cdot \partial \mathcal{H}_{L}\right)-i \frac{1}{2} \operatorname{Tr}\left(\overline{\mathcal{H}}_{R} v \cdot \partial \mathcal{H}_{R}\right) \\
-\frac{g_{\pi}}{4}\left[\operatorname{Tr}\left(\overline{\mathcal{H}_{L}} \Sigma^{\dagger} \mathcal{H}_{R}\right)+\operatorname{Tr}\left(\overline{\mathcal{H}_{R}} \Sigma \mathcal{H}_{L}\right)\right] \\
\quad-\Delta\left(\operatorname{Tr}\left(\overline{\mathcal{H}_{L}} \mathcal{H}_{L}\right)+\operatorname{Tr}\left(\overline{\mathcal{H}_{R}} \mathcal{H}_{R}\right)\right) \\
+i \frac{g_{A}}{2 f_{\pi}}\left[\operatorname{Tr}\left(\overline{\mathcal{H}_{L}} \gamma^{5}\left(/ \partial \Sigma^{\dagger}\right) \mathcal{H}_{R}\right)-\operatorname{Tr}\left(\overline{\mathcal{H}_{R}} \gamma^{5}(/ \partial \Sigma) \mathcal{H}_{L}\right)\right] \\
+\ldots
\end{gathered}
$$

The $\Delta$ term can be "gauged away" by a reparameterization transformation. In practice, we generalize this Lagrangian by transforming to a nonlinear realization of the fields and introduce a chiral parameter, $G_{A}$, which is equal to 1 in the linear realization.

The interplay between the chiral symmetry of the light quark and the spin-symmetry of the heavy quark had previously been explored in a simple dynamical model of heavy-light mesons by Bardeen and Hill[8] and by Nowak et al[9]. Approximating the color gluon interactions with a local current-current dynamics of a NambuJona-Lasinio model[10], the heavy-light meson spectrum can be determined and is related to the dynamical symmetry breaking in the light quark sector of the model. In this simple model, the heavy-light mesons do form supermultiplets and the dynamics can be described with an effective action of a linear sigma model.

From the motivation of this simple toy model, we developed an effective chiral Lagrangian to describe the tree-level interactions between the heavy-light mesons and the pseudoscalar mesons. There are couplings, $g_{A}$, that describe the intramultiplet pionic transitions, $D_{s}^{*} \rightarrow$ $D_{s}+\pi, D_{s J}^{*} \rightarrow D_{s}^{*}+\pi, D_{s J}^{*} \rightarrow D_{s J}+\pi$, and couplings, $G_{A}$, that describe the intramultiplet transitions, $D_{s J} \rightarrow D_{s}+\pi . g_{A}$ reflects the chirality of the constituent light quarks while $G_{A}$ reflects the chirality of the heavylight meson states. In a simple picture of linear chiral symmetry these couplings should be close to 1 , but they are free parameters for more general nonlinear realizations of chiral symmetry. Spin splittings arise from corrections to the static limit for the heavy quark.

Using the nonlinear chiral field, $\xi$, we can perform redefinitions of the heavy meson fields to bring them into linear flavor $S U(3)$ representations in the parity eigenbasis:

$$
\begin{gathered}
\Sigma=\xi \tilde{\sigma} \xi \quad \xi=\exp \left(i \tilde{\pi} / 2 f_{\pi}\right) \\
\mathcal{H}_{L}=\frac{1}{\sqrt{2}} \xi^{\dagger}\left(\mathcal{H}-i \mathcal{H}^{\prime}\right) \quad \mathcal{H}_{R}=\frac{1}{\sqrt{2}} \xi\left(\mathcal{H}+i \mathcal{H}^{\prime}\right)
\end{gathered}
$$

and the Lagrangian now takes the form:

$$
\begin{aligned}
\mathcal{L}_{L H}= & -\frac{1}{2} \operatorname{Tr}(\overline{\mathcal{H}} v \cdot(i \partial+\mathcal{V}) \mathcal{H})-\frac{1}{2} \operatorname{Tr}\left(\overline{\mathcal{H}}^{\prime} v \cdot(i \partial+\mathcal{V}) \mathcal{H}^{\prime}\right) \\
& +i \frac{1}{2} G_{A} \operatorname{Tr}\left(\overline{\mathcal{H}}^{\prime} v \cdot \mathcal{A} \mathcal{H}\right)-i \frac{1}{2} G_{A} \operatorname{Tr}\left(\overline{\mathcal{H}} v \cdot \mathcal{A} \mathcal{H}^{\prime}\right) \\
& +\frac{g_{\pi}}{4}\left[\operatorname{Tr}\left(\overline{\mathcal{H}}{ }^{\prime} \tilde{\sigma} \mathcal{H}^{\prime}\right)-\operatorname{Tr}(\overline{\mathcal{H}} \tilde{\sigma} \mathcal{H})\right] \\
+\frac{g_{A}}{2 f_{\pi}}[ & \left.\operatorname{Tr}\left(\overline{\mathcal{H}}^{\prime} \gamma^{5} \gamma_{\mu}\left\{\mathcal{A}^{\mu}, \tilde{\sigma}\right\} \mathcal{H}^{\prime}\right)-\operatorname{Tr}\left(\overline{\mathcal{H}} \gamma^{5} \gamma_{\mu}\left\{\mathcal{A}^{\mu}, \tilde{\sigma}\right\} \mathcal{H}\right)\right] \\
& +\frac{g_{A}}{2 f_{\pi}} \operatorname{Tr}\left(\overline{\mathcal{H}}^{\prime} \gamma^{5} \gamma_{\mu}\left(\partial^{\mu} \tilde{\sigma}-i\left[\mathcal{V}^{\mu}, \tilde{\sigma}\right]\right) \mathcal{H}\right) \\
& +\frac{g_{A}}{2 f_{\pi}} \operatorname{Tr}\left(\overline{\mathcal{H}} \gamma^{5} \gamma_{\mu}\left(\partial^{\mu} \tilde{\sigma}-i\left[\mathcal{V}^{\mu}, \tilde{\sigma}\right]\right) \mathcal{H}^{\prime}\right) \\
& +\ldots
\end{aligned}
$$

where:

$$
\begin{gathered}
\mathcal{V}_{\mu}=\frac{1}{2}\left(\xi^{\dagger} \partial_{\mu} \xi+\xi \partial_{\mu} \xi^{\dagger}\right)=\frac{i}{8 f_{\pi}^{2}}\left[\tilde{\pi}, \partial_{\mu} \tilde{\pi}\right]+\ldots \\
\mathcal{A}_{\mu}=i \frac{1}{2}\left(\xi^{\dagger} \partial_{\mu} \xi-\xi \partial_{\mu} \xi^{\dagger}\right)=-\frac{1}{2 f_{\pi}} \tilde{\pi}+\ldots
\end{gathered}
$$

and the scalar field, $\tilde{\sigma}$, has the vacuum expectation value, $<\tilde{\sigma}>=I_{3} f_{\pi}$.

Instanton effects in QCD break the $U(1)$ chiral symmetry of the massless quarks which has a significant impact on phenomenology of the scalar and pseudoscalar mesons. This chiral symmetry breaking is responsible for the large mass of the $\eta^{\prime}$ meson and has an important impact on the violation of the Zweig rule in the meson sector. In a linear sigma model description of the light mesons, a determinant term must be added to provide for the explicit breaking of the $U(1)$ chiral symmetry. This term generates the large $\eta \prime$ mass but has the opposite effect on the scalar boson spectrum where the octet scalars are heavy and the singlet scalar mass is suppressed. In our phenomenological analysis, we identify the scalar mesons with states above $1 \mathrm{GeV}$ consistent with the quenched lattice calculations of Bardeen et al[11], [12] implying that the lighter scalar mesons should be identified with four quark or two meson bound-states.

\section{DECAY PHENOMENOLOGY}

Since the masses of the new $D_{s} J$ mesons are below the corresponding strong thresholds, their strong decays are suppressed. The new states must decay through processes that violate the dominant strong interactions symmetries: (ISV) isospin violating, (ZRV) Zweig rule violating, or (EM) electromagnetic.

$$
\begin{array}{ll}
D_{s J}^{*}(2317) \rightarrow D_{s}(1968) \pi^{0} & \text { ISV, ZRV } \\
D_{s J}^{*}(2460) \rightarrow D_{s}(2112) \pi^{0} & \text { ISV, ZRV } \\
D_{s J}^{*}(2460) \rightarrow D_{s}(1968) \pi^{+} \pi^{-} & \text {ZRV } \\
D_{s J}^{*}(2317) \rightarrow D_{s}(2112) \gamma & \text { EM } \\
D_{s J}^{*}(2460) \rightarrow D_{s}(1968) \gamma & \text { EM } \\
D_{s J}^{*}(2460) \rightarrow D_{s}(2112) \gamma & \text { EM }
\end{array}
$$


TABLE I: The predicted hadronic and electromagnetic transistion rates for narrow $j_{l}^{P}=1 / 2^{-}(1 S)$ and $j_{l}^{P}=1 / 2^{+}(1 P)$ heavy-light states.

\begin{tabular}{|c|c|c|c|c|c|c|}
\hline system & transition & $\mathrm{Q}(\mathrm{keV})$ & overlap & dependence & $\Gamma(\mathrm{keV})$ & exptl BR \\
\hline \multirow[t]{3}{*}{$(c \bar{u})$} & $1^{-} \rightarrow 0^{-}+\gamma$ & 137 & 0.991 & $r_{\bar{c} u}$ & 33.5 & $(38.1 \pm 2.9) \%$ \\
\hline & $1^{-} \rightarrow 0^{-}+\pi^{0}$ & 137 & & $g_{A}$ & 43.6 & $(61.9 \pm 2.9) \%$ \\
\hline & total & & & & 77.1 & \\
\hline \multirow[t]{4}{*}{$(c \bar{d})$} & $1^{-} \rightarrow 0^{-}+\gamma$ & 136 & 0.991 & $r_{\bar{c} d}$ & 1.63 & $(1.6 \pm 0.4) \%$ \\
\hline & $1^{-} \rightarrow 0^{-}+\pi^{0}$ & 38 & & $g_{A}$ & 30.1 & $(30.7 \pm 0.5) \%$ \\
\hline & $1^{-} \rightarrow 0^{-}+\pi^{+}$ & 39 & & $g_{A}$ & 65.1 & $(67.7 \pm 0.5) \%$ \\
\hline & total & & & & 96.8 & $96 \pm 22$ \\
\hline \multirow[t]{3}{*}{$(c \bar{s})$} & $1^{-} \rightarrow 0^{-}+\gamma$ & 138 & 0.992 & $r_{\bar{c} s}$ & 0.43 & $(94.2 \pm 2.5) \%$ \\
\hline & $1^{-} \rightarrow 0^{-}+\pi^{0}$ & 48 & & $g_{A} \delta_{\eta \pi 0}$ & 0.0079 & $(5.8 \pm 2.5) \%$ \\
\hline & total & & & & 0.44 & \\
\hline \multirow[t]{3}{*}{$(c \bar{s})$} & $0^{+} \rightarrow 1^{-}+\gamma$ & 212 & 2.794 & $r_{\bar{c} s}$ & 1.74 & \\
\hline & $0^{+} \rightarrow 0^{-}+\pi^{0}$ & 297 & & $G_{A} \delta_{\eta \pi 0}$ & 21.5 & \\
\hline & total & & & & 23.2 & \\
\hline \multirow[t]{7}{*}{$(c \bar{s})$} & $1^{+} \rightarrow 0^{+}+\gamma$ & 138 & 0.992 & $r_{\bar{c} S}^{\prime}$ & 2.74 & \\
\hline & $1^{+} \rightarrow 0^{+}+\pi^{0}$ & 48 & & $g_{A} \delta_{\eta \pi 0}$ & 0.0079 & \\
\hline & $1^{+} \rightarrow 1^{-}+\gamma$ & 323 & 2.638 & $r_{\bar{c} s}$ & 4.66 & \\
\hline & $1^{+} \rightarrow 0^{-}+\gamma$ & 442 & 2.437 & $r_{\bar{c} s}$ & 5.08 & \\
\hline & $1^{+} \rightarrow 1^{-}+\pi^{0}$ & 298 & & $G_{A} \delta_{\eta \pi 0}$ & 21.5 & \\
\hline & $1^{+} \rightarrow 0^{-}+2 \pi$ & 221 & & $g_{A} \delta_{\sigma_{1} \sigma_{3}}$ & 4.2 & \\
\hline & total & & & & 38.2 & \\
\hline
\end{tabular}

The first two processes represent the dominant Goldberger-Treiman decays governed by the coupling, $G_{A}$, which is related to the spontaneous chiral symmetry breaking that generates the splitting between the opposite parity states. The small difference between the up and down quark masses is responsible for the isospin violation while the $U(1)$ chiral symmetry breaking is required for the Zweig rule violation needed for the transition between a state containing only strange and charm quarks to a state including additional up and down quarks. The third process also requires a Zweig rule violation to produce the light pions in the final state. In this case, the decay proceeds through the emission of virtual scalar boson and requires the singlet-octet mixing generated by the $U(1)$ symmetry breaking. This decay depends on the coupling, $g_{A}$, which also determines the intramultiplet pionic transitions.

The radiative decays involve both $E 1$ and $M 1$ electromagnetic transitions. These are calculated using potential model wavefunctions for the heavy-light mesons. For the $\bar{c} s$ states, there is a significant cancellation due to the heavy quark mass and charge. Using constituent quark masses, the suppression factor for the above electromagnetic decay amplitudes is given by $r_{\bar{c} s}=(1-$ $\left.m_{s} e_{s} / m_{\bar{c}} e_{\bar{c}}\right) \sim 0.38$ contrary to the static limit where $r_{\bar{c}_{s}}=1$. For the analogous b-quark states this factor would yield a modest enhancement of the amplitudes for $B_{s J}$ decays.
We use the usual constituent quark potential model to estimate the electromagnetic transition rates. For the M1 magnetic transitions $1^{-} \rightarrow 0^{-} \gamma$ the rate is given by:

$$
\Gamma_{\mathrm{M} 1}(i \rightarrow f \gamma)=\frac{4 \alpha}{3} \mu_{\bar{Q} q}^{2} k^{3}\left(2 J_{f}+1\right)\left|\left\langle f\left|j_{0}(k r)\right| i\right\rangle\right|^{2},
$$

where the magnetic dipole moment is:

$$
\mu_{\bar{Q} q}=\frac{m_{Q}^{*} e_{q}-m_{q}^{*} e_{\bar{Q}}}{2 m_{Q}^{*} m_{q}^{*}}=\frac{e_{q}}{2 m_{q}^{*}} r_{\bar{Q} q}
$$

and $k$ is the photon energy.

The strength of the electric-dipole transitions is governed by the size of the radiator and the charges of the constituent-quarks. The E1 transition rate is given by

$\Gamma_{\mathrm{E} 1}(i \rightarrow f+\gamma)=\frac{\left.4 \alpha<e_{\mathrm{avg}}\right\rangle^{2}}{27} k^{3}\left(2 J_{f}+1\right)|\langle f|r| i\rangle|^{2} \mathcal{S}_{i f}$,

where the mean charge is

$$
<e_{\mathrm{avg}}>=\frac{m_{Q}^{*} e_{q}-m_{q}^{*} e_{\bar{Q}}}{m_{Q}^{*}+m_{q}^{*}}=\frac{e_{q} m_{Q}^{*} r_{\bar{Q} q}}{m_{Q}^{*}+m_{q}^{*}},
$$

$k$ is the photon energy, and the statistical factor, $\mathcal{S}_{i f}$, for $(i, f)=\left(0^{+}, 1^{-}\right)$is 1 , for $\left(1^{+}, 1^{-}\right)$is $2 / 3$, and for $\left(1^{+}, 0^{-}\right)$ is 1 .

For the $1^{+} \rightarrow 0^{+} \gamma$ M1 transition we define the coefficient $r_{\bar{Q} q}^{\prime}$ :

$$
r_{\bar{Q} q}^{\prime}=\left(1+3 \frac{m_{q}^{*} e_{\bar{Q}}}{m_{\bar{Q}}^{*} e_{q}}\right)
$$


TABLE II: Comparison of $D_{s J}(2460)$ branching ratios.

\begin{tabular}{ccc}
\hline \hline Branching Ratio & Babar & BEH \\
\hline$B\left(D_{s J}(2460) \rightarrow D_{s}^{*} \pi^{0}\right.$ & $56 \pm 13 \pm 9 \%$ & $56 \%$ \\
$B\left(D_{s J}(2460) \rightarrow D_{s}^{*} \gamma\right.$ & $16 \pm 4 \pm 3 \%$ & $13 \%$ \\
$B\left(D_{s J}(2460) \rightarrow D_{s}^{*} \gamma\right.$ & $4 \pm 1 \%$ & $5 \%$ \\
\hline \hline
\end{tabular}

The decay rate is given by:

$$
\Gamma_{\mathrm{M} 1}(i \rightarrow f \gamma)=\frac{4 \alpha}{3} \mu_{\bar{Q} q}^{\prime 2} k^{3}\left(2 J_{f}+1\right)\left|\left\langle f\left|j_{0}(k r)\right| i\right\rangle\right|^{2},
$$

where the effective magnetic dipole moment $\mu_{\bar{Q} q}^{\prime}$ is now:

$$
\mu_{\bar{Q} q}^{\prime}=\frac{-m_{Q}^{*} e_{q}-3 m_{q}^{*} e_{\bar{Q}}}{6 m_{Q}^{*} m_{q}^{*}}=-\frac{e_{q}}{6 m_{q}^{*}} r_{\bar{Q} q}^{\prime}
$$

and $k$ is the photon energy,

$$
r_{\bar{c} s}^{\prime}=2.88 \quad r_{\bar{b} s}^{\prime}=0.70 .
$$

The predictions of Bardeen, Eichten and Hill[7] for the decays of the $D_{s}$ chiral supermultiplet states are shown in Table I. The category "Overlap" is the reduced matrix element overlap integral and "dependence" refers to sensitivity to the model parameters listed. We take $G_{A}=1$ and extract $g_{A}$ from a fit to the $D^{+*}$ total width. Note that the $\bar{c} s$ transitions are sensitive to $r_{\bar{c} s}$; if we implement the observed ratio of branching fractions $\left(D_{s}\left(1^{-}\right) \rightarrow D_{s}\left(0^{-}\right) \pi^{0}\right) / \Gamma\left(D_{s}\left(1^{-}\right) \rightarrow D_{s}\left(0^{-}\right) \gamma\right)=$ $0.062 \pm 0.026$ then the E1 radiative transitions for the $\bar{c} s$ system should be reduced by a factor of $\sim 3$. The electromagnetic transitions are somewhat suppressed compared to the dominant isospin violating pionic transitions. The Zwieg rule violating transitions to two pions make an important contribution to the decay of the $D_{s J}(2460)$.

The most direct comparison of our results with experiment comes from the studies of $D_{s J}$ mesons produced directly in the decays of B-mesons by Babar[13] and Belle[14]. The experiments have determined the absolute branching ratios for the some of the $D_{s J}(2460)$ decays. In Table 2 we show the comparison of the Babar results[15] with our predictions. In Table 3 we give the results of a comprehensive study by Babar[16] of branching ratios for the decays of $D_{s J}$ mesons in inclusive reactions.

The results seem to be in general agreement with our predictions confirming the identification of the new $D_{s J}$ states as chiral partners to the $D_{s}$ ground-states and the $(\bar{c} s)$ constituent quark picture of the bound-states. Similar results have been subsequently been obtained by Godfrey[17] and by Colangelo, et al[18]. Their fits prefer a somewhat smaller value of $G_{A}, G_{A} \sim 0.7$, but are still consistent with our general picture. The mass splitting between the opposite parity heavy-light mesons has also been discussed by Deandrea, et al[19] and by Nowak, et al[20].
TABLE III: A summary of branching-ratio results. The first quoted uncertainty for the central value is statistical and the second is systematic. The limits correspond to 95\% CL. For the hypothetical $D_{s J}^{*}(2317)^{++}$and $D_{s J}^{*}(2317)^{0}$ mesons, an unknown additional factor from the ratio of production

\begin{tabular}{|c|c|c|c|}
\hline Decay Mode & Centr & ral Value & Limit \\
\hline \multicolumn{4}{|c|}{$\mathcal{B}\left(D_{s J}^{*}(2317)^{+} \rightarrow X\right) / \mathcal{B}\left(D_{s J}^{*}(2317)^{+} \rightarrow D_{s}^{+} \pi^{0}\right)$} \\
\hline$D_{s}^{+} \gamma$ & $-0.02 \pm$ & $0.02 \pm \quad 0.08$ & $<0.14$ \\
\hline$D_{s}^{+} \pi^{0} \pi^{0}$ & $0.08 \pm$ & $0.06 \pm$ & $<0.25$ \\
\hline$D_{s}^{+} \gamma \gamma$ & $0.06 \pm$ & $0.04 \pm$ & $<0.18$ \\
\hline$D_{s}^{*}(2112)^{+} \gamma$ & $0.00 \pm$ & $0.03 \pm$ & $<0.16$ \\
\hline$D_{s}^{+} \pi^{+} \pi^{-}$ & $0.0023 \pm 0$. & $.0013 \pm 0.0002$ & $<0.0050$ \\
\hline \multicolumn{4}{|c|}{$\mathcal{B}\left(D_{s J}(2460)^{+} \rightarrow X\right) / \mathcal{B}\left(D_{s J}(2460)^{+} \rightarrow D_{s}^{+} \pi^{0} \gamma\right)^{a}$} \\
\hline$D_{s}^{+} \pi^{0}$ & $-0.023 \pm$ & $0.032 \pm 0.005$ & $<0.042$ \\
\hline$D_{s}^{+} \gamma$ & $0.337 \pm$ & $0.036 \pm 0.038$ & - \\
\hline$D_{s}^{*}(2112)^{+} \pi^{0}$ & $0.97 \pm$ & $0.09 \pm$ & $>0.75$ \\
\hline$D_{s J}^{*}(2317)^{+} \gamma$ & $0.03 \pm$ & $0.09 \pm$ & $<0.25$ \\
\hline$D_{s}^{+} \pi^{0} \pi^{0}$ & $0.13 \pm$ & $0.13 \pm$ & $<0.68$ \\
\hline$D_{s}^{+} \gamma \gamma$ & $0.08 \pm$ & $0.10 \pm$ & $<0.33$ \\
\hline$D_{s}^{*}(2112)^{+} \gamma$ & $-0.02 \pm$ & $0.08 \pm$ & $<0.24$ \\
\hline$D_{s}^{+} \pi^{+} \pi^{-}$ & $0.077 \pm$ & $0.013 \pm 0.008$ & - \\
\hline \multicolumn{4}{|c|}{$\sigma\left(D_{s J}^{*}(2317)^{++}\right) / \sigma\left(D_{s J}^{*}(2317)^{+}\right) \times$} \\
\hline \multicolumn{4}{|c|}{$\mathcal{B}\left(D_{s J}^{*}(2317)^{++} \rightarrow X\right) / \mathcal{B}\left(D_{s J}^{*}(2317)^{+} \rightarrow D_{s}^{+} \pi^{0}\right)$} \\
\hline$D_{s}^{+} \pi^{+}$ & & - & $<0.017$ \\
\hline \multicolumn{4}{|c|}{$\sigma\left(D_{s J}^{*}(2317)^{0}\right) / \sigma\left(D_{s J}^{*}(2317)^{+}\right) \times$} \\
\hline \multicolumn{4}{|c|}{$\mathcal{B}\left(D_{s J}^{*}(2317)^{0} \rightarrow X\right) / \mathcal{B}\left(D_{s J}^{*}(2317)^{+} \rightarrow D_{s}^{+} \pi^{0}\right)$} \\
\hline$D_{s}^{+} \pi^{-}$ & & - & $<0$ \\
\hline
\end{tabular}
cross sections is involved. A lower limit is quoted for the $D_{s J}(2460)^{+} \rightarrow D_{s}^{*}(2112)^{+} \pi^{0}$ results.

${ }^{a}$ Denominator includes both $D_{s}^{*}(2112)^{+} \pi^{0}$ and $D_{s J}^{*}(2317)^{+} \gamma$ channels.

\section{CONCLUSIONS}

The discovery of novel narrow states of heavy-light mesons by Babar, Cleo and Belle provides us with a new window on quantum chromodynamics and the opportunity to explore the nature of the chiral symmetry of the light quark. Heavy-light mesons are the simplest boundstates containing a single light constituent quark. Combining the heavy quark symmetry of the charm quark and the light quark chiral symmetry, we have identified the new $D_{s J}$ states and the $D_{s}$ ground-states as members of a chiral supermultiplet and have achieved a reasonable description of their decays.

Our original picture of the mass-splitting between the opposite parity partners involved a partial suppression of the spontaneous chiral symmetry breaking of the light quark in the presence of the field of the heavy quark. It now seems likely that part of the explanation for the observed mass-splitting is related to threshold effects coming from the strong "Goldberger-Treiman" coupling to virtual states with two mesons. In this sense, the new states can also be viewed as having a four-quark or two 
meson component.

\section{ACKNOWLEDGMENTS}

This talk will appear in the proceedings of the Chiral '07 Workshop held November 13-16, 2007 hosted by
RCNP, Osaka University. I would like to thank Estia Eichten and Chris Hill for help in preparing this manuscript. I also thank the Yukawa Institute for Theoretical Physics for its hospitality and support during my stay in Japan. This manuscript has been authored by the Fermi Research Alliance LLC under Contract No. DEAC0207CH11359 with the U.S. Department of Energy.
[1] [Babar], B. Aubert, et al, Phys.Rev.Lett. 90:242001 (2006), hep-ex/0304021.

[2] [Cleo], D. Besson, et al, Phys.Rev. D68:032002 (2003), hep-ex/0305100.

[3] [Belle], Y. Mikami, et al, Phys.Rev.Lett. 92:012002 (2004), hep-ex/0307052

[4] S. Godfrey and N. Isgur, Phys.Rev. D32,189 (1985).

[5] S. Godfrey and R. Kokoski, Phys.Rev. D43,1679 (1991).

[6] M. Di Pierro and E. Eichten, Phys.Rev. D64:114004 (2002).

[7] W. Bardeen, E. Eichten, C. Hill, Phys.Rev. D68:054024 (2003), hep-ph/0305049.

[8] W. Bardeen and C. Hill, Phys.Rev. D49,409 (1994).

[9] M. Nowak, M. Rho and I. Zahed, Phys.Rev. D48,4370 (1993).

[10] Y. Nambu and G. Jona-Lasinio, Phys.Rev. 122,345 (1961); ibid. 124,246 (1961).

[11] W. Bardeen, et al, Phys.Rev. D65:014509 (2001), hep- lat/0106008.

[12] W. Bardeen, et al, Phys.Rev. D69:054502 (2004), heplat/0307023.

[13] [Babar], B. Aubert, et al, Phys.Rev.Lett 93:181801 (2004), hep-ex/0408041.

[14] [Belle], K. Abe, et al, 2005 Lepton-Photon Conference, hep-ex/0507064.

[15] [Babar], B. Aubert, et al, Phys.Rev. D74:031103 (2006), hep-ex/0605036.

[16] [Babar], B. Aubert, et al, Phys.Rev. 032007 (2006), hepex/0604030.

[17] S. Godfrey, Phys.Lett. B568:254 (2003).

[18] P. Colangelo, et al, Phys.Lett. B570:180 (2003).

[19] A. Deandrea, et al, Phys.Rev. D68:097501 (2003).

[20] M. Nowak, M. Rho and I. Zahed, Acta.Phys.Polon. B35:2377 (2004). 